\title{
Clinical characteristics in urolithiasis formation according to body mass index
}

\author{
HISASHI TAKEUCHI and TEIICHIRO AOYAGI \\ Department of Urology, Tokyo Medical University Ibaraki Medical Center, Ibaraki 300-0395, Japan
}

Received October 19, 2018; Accepted June 5, 2019

DOI: $10.3892 /$ br.2019.1220

\begin{abstract}
Although urolithiasis is considered to be strongly associated with lifestyle habits, there are numerous cases in which urolithiasis develops despite a non-obese body type or healthy lifestyle habits. However, in clinical practice, the diet therapy and lifestyle changes instructed for the prevention of recurrence of urolithiasis are almost identical in numerous cases. Therefore, the present study examined the effect of body mass index (BMI) on urolithiasis and its surrounding environment in patients, by analyzing the number of normal- and high-BMI (healthy and overweight) patients with urolithiasis. The present study analyzed 63 patients with urolithiasis for whom height and weight were measured at our hospital (Tokyo Medical University Ibaraki Medical Center). BMI $<25$ represents healthy body types and BMI $\geq 25$ BMI defines overweight body types. Thus, patients were then grouped by BMI-defined body type using a threshold value of 25 accordingly. It was observed that a higher percentage of males were obese compared with females. Upon comparing the normaland high-BMI groups, no significant difference was observed in uric acid level, urine $\mathrm{pH}$ or calculus number between the two groups. Liver computed tomography (CT) values were significantly lower in the high-BMI group compared with the normal-BMI group. There was no significant correlation between calculus size counts and BMI. However, a significant negative correlation was observed between BMI and the liver CT value. These results suggest that liver CT values correlated negatively with BMI, but the data indicates that other mechanisms unassociated with a fatty liver may be involved in urolithiasis in non-obese patients. The results of the present study suggest that physicians should consider the mechanism involved in preventing the recurrence of urolithiasis.
\end{abstract}

Correspondence to: Dr Hisashi Takeuchi, Department of Urology, Tokyo Medical University Ibaraki Medical Center, 3-20-1 Chuo, Ami-machi, Inashiki-gun, Ibaraki 300-0395, Japan

E-mail: hisashi917@yahoo.co.jp

Key words: urolithiasis formation, body mass index, gender differences, urinary stone disease, lifestyle disease

\section{Introduction}

Urolithiasis is one of the most prevalent urological diseases in the nineteenth century population in Europe is quite similar to that of the twentieth century in Asia (1). The incidence of urolithiasis in Japan has steadily increased between 1965 and 2005 (2). Urolithiasis has been reported to increase the number of patients of various dietary habits. It has been reported that $12 \%$ of people globally suffer from urolithiasis (3). The increase in urolithiasis prevalence is thought to associated with changes in dietary habits, but may be particularly associated with a higher intake of animal protein $(4,5)$.

Urolithiasis is a metabolic syndrome that presents symptoms including hypertension, lipid metabolism disorders, diabetes, obesity, atherosclerosis, ischemic heart disease and other associated problems that have a common pathology of visceral fat accumulation and insulin resistance (6-10). This common pathology means urolithiasis is considered to be a lifestyle disease. Various studies have reported cases of urolithiasis associated with hyperlipidemia, obesity, fatty liver, hyperuricemia, diabetes, hypertension and aortic calcification $(9,10)$

Although urolithiasis is considered to be strongly associated with lifestyle habits, there are numerous cases in which urolithiasis develops despite a non-obese body type or healthy lifestyle habits (6-10). However, in clinical practice, diet therapy and lifestyle changes instructed for the prevention of recurrence of urolithiasis are almost identical in numerous different cases.

Therefore, the present study examined the effect of body mass index (BMI) on urolithiasis and its surrounding environment in patients, by analyzing the number of normal- and high-BMI (healthy and overweight) patients with urolithiasis. Based on their BMI, patients with urolithiasis were categorized into a standard-type patient group and an obese-type patient group, and differences in clinical factors were assessed.

\section{Materials and methods}

The present study analyzed a total of 63 patients with urolithiasis for whom height and weight were measured at our hospital (Tokyo Medical University Ibaraki Medical Center, Ibaraki, Japan) between July 2013 and June 2015. The mean 
age of the patients was 55.6 years (range, 59-78 years). Of the total 63 patients, 49 were male and 14 were female (Table I). Written informed consent was obtained from all patients and all procedures used in the present study were ethically approved by the Ethical Committee of our hospital (Tokyo Medical University Ibaraki Medical Center). For each patient, BMI was calculated initially. According to World Health Organization guidelines, BMI $<25$ is of healthy body types and BMI $\geq 25$ represents overweight body types (11). Thus, patients were then grouped by BMI-defined body type using a threshold value of 25 accordingly (11).

The measurement of the computed tomography (CT) value was determined using the Hounsfield Unit (HU). The liver CT value was defined as the mean value of three $\mathrm{CT}$ values selected randomly. Two CT model types, the Brilliance iCT SP 64-Slice configuration (Philips Healthcare, Amsterdam, The Netherlands) and the SOMATOM Sensation 64-Slice configuration (Siemens AG, Munich, Germany) were used.

Statistical analysis. All statistical analyses were performed using Stat View (ver. 5.0; SAS Institute, Inc., Cary, NC, USA). Continuous variables were presented as the mean \pm standard deviation. A $\chi^{2}$ test was used to evaluate comparisons between the differences in sex between the $\mathrm{BMI} \geq 25$ and $\mathrm{BMI}<25$ groups (Table II). A Student's t-test was used to evaluate the comparisons between the clinical features, including BMI, calculus size, serum uric acid value, urinary $\mathrm{pH}$ value and liver CT value (Fig. 1). The correlations amongst the liver CT value, calculus size and BMI were determined using a Spearman's rank correlation test (Fig. 2). $\mathrm{P}<0.05$ was considered to indicate a statistically significant difference. The difference between the mean values of the two groups was analyzed using a Student's t-test, and the difference between the two variables was analyzed using a $\chi^{2}$ test. Regression analysis was used to identify correlations between variables.

\section{Results}

The clinical features of the 63 patients with urolithiasis are listed in Table I. The mean age of the patients was 55.6 years (range, 59-78 years). Of the total 63 patients, 49 were male and 14 were female. The mean BMI was 24.4 (range, 17.6-41.0). The mean value of the urine PH was 6.63 (range, 5.5-7.5). The mean value of the calculus size was $9.5 \mathrm{~mm}$ (range, 4-35). The mean value of the uric acid was $5.86 \mathrm{mg} / \mathrm{dl}$ (range, 2.3-10.1). The mean value of the liver CT was 56.2 (range, 30-70).

The comparison of the BMI between males and females revealed that the male patients with urolithiasis had a higher BMI compared with the female patients with urolithiasis. It was observed that there was a higher percentage of obesity amongst males compared with females, however this difference was not significant ( $\mathrm{P}=0.2207$; Table II).

Association amongst clinical characteristics and BMI in patients with urolithiasis. Upon comparing the normal- and high-BMI groups, no significant differences were observed in uric acid level $(\mathrm{P}=0.1836$; Fig. $1 \mathrm{~A})$, urine $\mathrm{PH}(\mathrm{P}=0.4073$;
Fig. 1B) or calculus size ( $\mathrm{P}=0.8129$; Fig. 1D) between the two groups. However, liver CT values were significantly lower in the high-BMI group compared with the normal-BMI group. $(\mathrm{P}=0.001$; Fig. 1C).

Correlation between liver CT value, calculus size counts and BMI in patients with urolithiasis. There was no significant correlation between calculus size counts and $\mathrm{BMI}(\mathrm{P}=0.9176$; Fig. 2A). However, a significant negative correlation was observed between BMI and liver CT value $(\mathrm{P}=0.0004$; Fig. 2B).

\section{Discussion}

The present study examined whether there exist differences in a number of variables, including body type and BMI, amongst patients with urolithiasis. The present study examined patients with urolithiasis, identified the differences between obese-type patients with urolithiasis and normal-type patients, and deduced that the CT value of the liver in obese-type patients with urolithiasis is significantly lower compared with that in standard-type patients with urolithiasis, and that obese-type urolithiasis is more common in males compared with in females. However, these were no significant differences between obese-type patients with urolithiasis and standard-type patients with urolithiasis in other factors other than liver CT values. The present study revealed that obese-type patients with urolithiasis were more common amongst males, and standard-type patients with urolithiasis were more common amongst females; and these results suggest that lifestyle-associated disease factors may not be involved in the formation of urolithiasis in females. Furthermore, these results suggest that the prevention of recurrence in females with urolithiasis may be less useful compared with that in males.

The present data indicated that liver CT values were significantly lower in obese patients (high-BMI) with urolithiasis. In abdominal CT examination, the liver CT value decreases with fat deposition in such a manner that $\mathrm{CT}$ value has been noted to be associated with fatty liver (12). In fact, fatty liver may be diagnosed using the CT value of the liver (13). The results of the present study suggest that only fatty liver, amongst all the symptoms of metabolic syndrome, was significantly different between normal- and high-BMI groups.

Fatty liver affects not only the liver, but also other aspects of metabolism. 'Fatty liver is a phenotype in the liver of metabolic syndrome' (14) and is closely associated with insulin resistance (15). Furthermore, it has been reported to be a predictor of coronary artery disease independent of other risk factors (16).

An increase in urolithiasis has been associated with an increase in metabolic syndrome (6-10). In particular, associations with type 2 diabetes, a high BMI, hypertension, hyperlipidemia (6) and cardiovascular diseases have been noted (7). It has been reported that the decrease in urine $\mathrm{pH}$ resulting from metabolic syndrome is the mechanism for the increase in urolithiasis (10). Increased acid excretion through urine and ammonium excretion disorder are considered to be responsible for decreased urinary $\mathrm{pH}$; in patients with diabetes, the main cause of uric acid 
Table I. Clinical features of the 63 patients with urolithiasis.

Characteristics

Value

Age, mean (range)

Sex, n

Body Mass Index, mean (range)

Size of urolithiasis, mean (range)

Number of urolithiasis, $\mathrm{n}$

Value of uric acid, mean (range)

Value of urine $\mathrm{pH}$, mean (range)

Value of liver computed tomography, mean (range)
55.6 years (range, $59-78$ years)

Male 49, female 14

$24.4(17.6-41.0)$

$9.5 \mathrm{~mm}(4-35)$

Single 36 , double 5 , more than triple 22 $5.86 \mathrm{mg} / \mathrm{dl}(2.3-10.1)$

$6.63(5.5-7.5)$

56.2 Hounsfield Unit (30-70)

$\mathrm{n}$, number of patients.

Table II. Comparison of BMI between male and female patients with urolithiasis.

\begin{tabular}{lccc}
\hline Gender & BMI $<25$ & BMI $\geq 25$ & Rate of high BMI $(\%)$ \\
\hline Male $(n=49)$ & 26 & 23 & 46.9 \\
Female $(n=14)$ & 10 & 4 & 28.6 \\
Total $(n=63)$ & 36 & 27 & 42.9 \\
\hline
\end{tabular}

n, number of patients; BMI, body mass index.
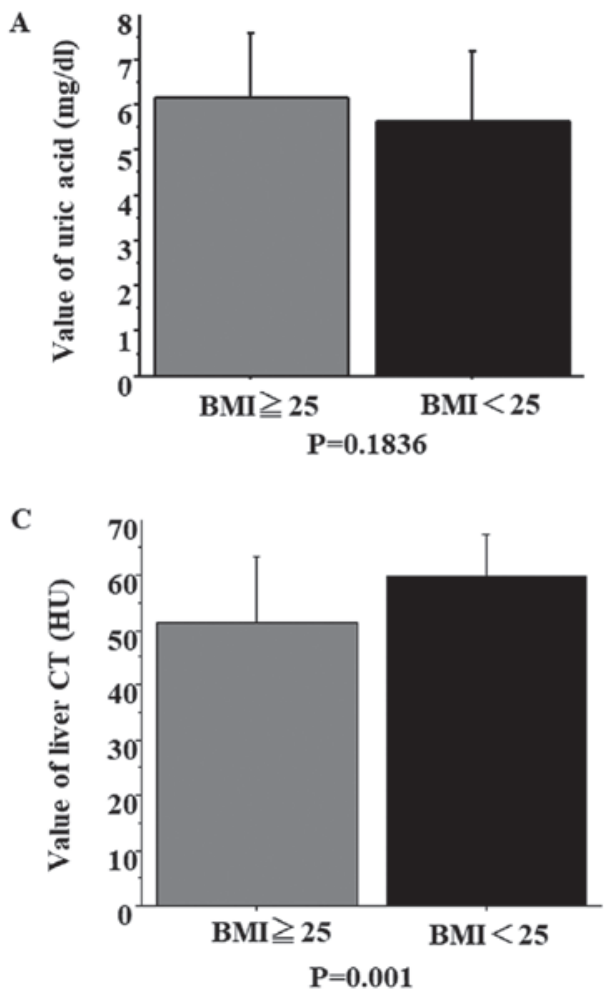
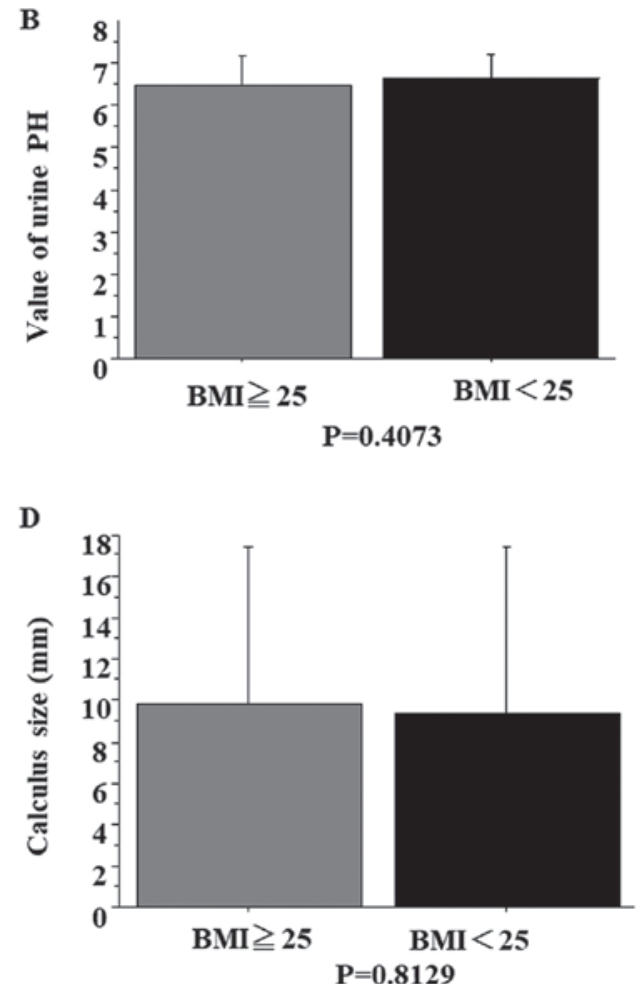

Figure 1. Comparisons between clinical characteristics and BMI in patients with urolithiasis. Continuous variables were presented as the mean \pm standard deviation. (A) Comparison of the value of uric acid. (B) Comparison of the value of urine pH. (C) Comparison of the value of liver CT. (D) Comparison of calculus size. BMI, body mass index; CT, computed tomography; HU, Hounsfield Unit.

calculus, a common complication, is low urine $\mathrm{pH}$ (10). One study suggests that an increased intake of fructose causes metabolic syndrome, and the low urinary $\mathrm{pH}$ in turn results in an increase in urolithiasis (17). In particular, urine $\mathrm{pH}$ is 

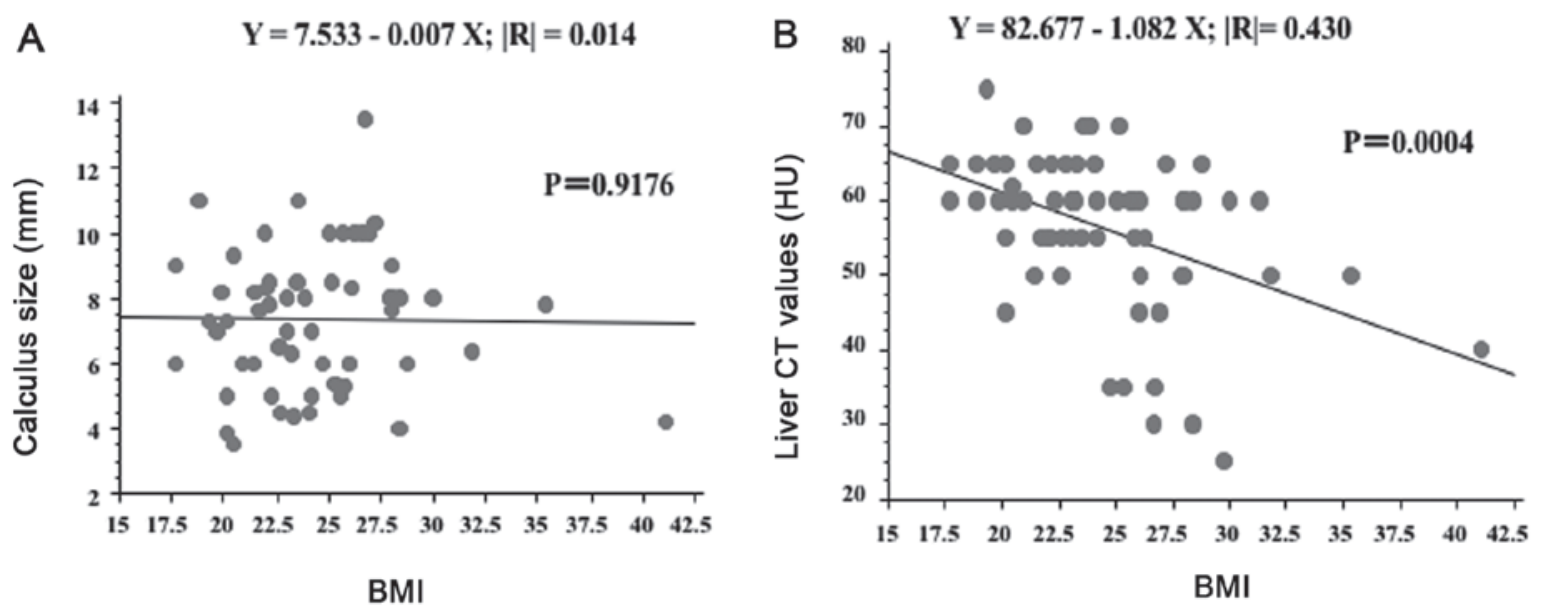

Figure 2. Correlation between liver CT value, calculus size counts and BMI in patients with urolithiasis. (A) Correlation between calculus size counts and BMI. (B) Correlation between liver CT value and BMI. CT, computed tomography; BMI, body mass index; HU, Hounsfield Unit.

persistently low (usually there is a diurnal variation with a transient rise in urine $\mathrm{pH}$ during the daytime). The diurnal variation of urine $\mathrm{pH}$ disappears in urolithiasis, uric acid becomes insoluble and is precipitated, and uric acid stones are formed (18).

A report on urolithiasis and metabolic syndrome-associated factors from Japan noted that fatty liver in male patients is a notable risk factor, an observation that the present study supports (19). However, in a large-scale cohort study in the United States it was observed that weight and BMI are associated with the onset of calculus in males and females, and that this association is stronger in females (20). In Japan, detailed cross-sectional studies on the presence or absence of insulin resistance were performed, and they determined that female patients with kidney stones had high insulin resistance and insulin values, supporting the link between obesity and urolithiasis (21). Furthermore, one study reports that urolithiasis formation (calcium phosphate) is observed in the kidney when rats receive a high cholesterol diet (22).

It has been reported that urolithiasis is similar to arteriosclerotic lesions, one of the metabolic syndromes, in cross-sectional studies and studies using mice models. In addition, in this phenomenon, the number of macrophages $(\mathrm{M} \varphi)$ of the renal interstitium increased with the formation of calculus and an image of $\mathrm{M} \varphi$ phagocytizing the crystal was observed (23). From these results of this previous study, the formation and disappearance of nephrolithiasis may be mostly attributed to the adhesion of crystals to the renal tubule cells, which are then transferred to the interstitial crystalline mass, the expression of chemokines/cytokines and other associated processes (23). Subsequent to adhesion to vascular endothelial cells, M $\varphi$ mature, begin phagocytosis, intracellular digestion, antigen presentation of $\mathrm{M} \varphi$ and finally digest the crystal (24). However, M1 macrophages are pro-inflammatory, and M2 anti-inflammatory (25); M1s are associated with adipocytes and metabolic syndrome, whereas M2s are associated with organ restoration $(26,27)$ and carcinogenesis (28). When crystals adhere to the renal tubular epithelium, M1-associated genes are highly expressed and promoted by adipocytes (29); in mice in which M2 macrophages are dysfunctional, M1 macrophages and kidney stone formation are increased (30).
There may be a number of potential limitations in the present study. Although the present study focused on BMI, urolithiasis is a multifactorial disease. Additionally, although using BMI as a marker is not the best method to measure obesity, it has been focused on in this paper as limited other studies on urolithiasis, to date, have focused on it.

Further studies, including studies with a larger number of cases, a greater number of clinical factors included, and the time course of treatment progress being taken into consideration, are warranted to rigorously examine the characteristics associated with urolithiasis formation.

Liver CT values correlated negatively with BMI, but the present data indicate that other mechanisms unassociated with fatty liver may be involved in urolithiasis in non-obese patients. It may be suggested that physicians should consider the mechanism involved in preventing the recurrence of urolithiasis.

\section{Acknowledgements}

Not applicable.

\section{Funding}

No funding was received.

\section{Availability of data and materials}

Not applicable.

\section{Authors' contributions}

HT designed the study, and wrote the initial draft of the manuscript. HT contributed to analysis and interpretation of data, and assisted in the preparation of the manuscript. HT contributed to data collection and interpretation, and critically reviewed the manuscript. All authors approved the final version of the manuscript, and agree to be accountable for all aspects of the work in ensuring that questions related to the accuracy or integrity of any part of the work are appropriately investigated and resolved. 


\section{Ethics approval and consent to participate}

All procedures used in this research were approved by the Ethical Committee of Tokyo Medical University Ibaraki Medical Center. Written informed consent was obtained from all patients.

\section{Patient consent for publication}

Not applicable.

\section{Competing interests}

The authors declare that they have no competing interests.

\section{References}

1. Asper R: Epidemiology and socioeconomic aspects of urolithiasis. Urol Res 12: 1-5, 1984.

2. Yasui T, Iguchi M, Suzuki S and Kohri K: Prevalence and epidemiological characteristics of urolithiasis in Japan: National trends between 1965 and 2005. Urology 71: 209-213, 2008.

3. Pak CY: Kidney stones. Lancet 351: 1797-1801, 1998.

4. Breslau NA, Brinkley L, Hill KD and Pak CY: Relationship of animal protein-rich diet to kidney stone formation and calcium metabolism. J Clin Endocrinol Metab 66: 140-146, 1988.

5. Curhan GC, Willett WC, Rimm EB and Stampfer MJ: A prospective study of dietary calcium and other nutrients and the risk of symptomatic kidney stones. N Engl J Med 328: 833-838, 1993.

6. Sakhaee K: Nephrolithiasis as a systemic disorder. Curr Opin Nephrol Hypertens 17: 304-309, 2008.

7. Sakhaee K and Maalouf NM: Metabolic syndrome and uric acid nephrolithiasis. Semin Nephrol 28: 174-180, 2008.

8. Cameron MA, Maalouf NM, Adams-Huet B, Moe OW and Sakhaee K: Urine composition in type 2 diabetes: Predisposition to uric acid nephrolithiasis. J Am Soc Nephrol 17: 1422-1428, 2006

9. Abate N, Chandalia M, Cab-Chan AV Jr, Moe OW and Sakhaee K: The metabolic syndrome and uric acid nephrolithiasis: Novel features of renal manifestation of insulin resistance. Kidney Int 65: 386-392, 2004.

10. Maalouf NM, Cameron MA, Moe OW, Adams-Huet B and Sakhaee K: Low urine pH: A novel feature of the metabolic syndrome. Clin J Am Soc Nephrol 2: 883-888, 2007.

11. WHO Expert Consultation: Appropriate body-mass index for Asian populations and its implications for policy and intervention strategies. Lancet 363: 157-163, 2004.

12. Bydder GM, Kreel L, Chapman RW, Harry D, Sherlock S and Bassan L: Accuracy of computed tomography in diagrosis of fatty liver. Br Med J 281: 1042, 1980.

13. Kan H, Kimura Y, Hyogo H, Fukuhara T, Fujino H, Naeshiro N, Honda Y, Kawaoka T, Tsuge M, Chayama K, et al: Non-invasive assessment of liver steatosis in non-alcoholic fatty liver disease. Hepatol Res 44: E420-E427, 2014.

14. Marchesini G, Brizi M, Bianchi G, Tomassetti S, Bugianesi E, Lenzi M, McCullough AJ, Natale S, Forlani G and Melchionda N: Nonalcoholic fatty liver disease: A feature of the metabolic syndrome. Diabetes 50: 1844-1850, 2001.
15. Maruhama Y, Ohneda A, Tadaki H, Ohtsuki M and Yanbe A Hepatic steatosis and the elevated plasma insulin level in patients with endogenous hypertriglyceridemia. Metabolism 24: 653-664, 1975.

16. Targher G, Bertolini L, Rodella S, Tessari R, Zenari L, Lippi G and Arcaro G: Nonalcoholic fatty liver disease is independently associated with an increased incidence of cardiovascular event in type 2 diabetic patients. Diabetes Care 30: 2119-2121, 2007.

17. Taylor EN and Curhan GC: Fructose consumption and the risk of kidney stones. Kidney Int 73: 207-212, 2008.

18. Murayama T, Sakai N, Takano T and Yamada T: Role of the diurnal variation of urinary $\mathrm{pH}$ and urinary calcium in urolithiasis: A study in outpatients. Int J Urol 8: 525-532, 2001.

19. Sakai N: Recent issues in urolithiasis. J Analytical Bio Sci 32: 215-219, 2009.

20. Taylor EN, Stampfer MJ and Curhan GC: Obesity, weight gain, and the risk of kidney stones. JAMA 293: 455-462, 2005.

21. Ando R, Suzuki S, Nagaya T, Yamada T, Okada A, Yasui T, Tozawa K, Tokudome S and Kohri K: Impact of insulin resistance, insulin and adiponectin on kidney stones in the Japanese population. Int J Urol 18: 131-138, 2011.

22. Strohmaier WL, Witte B and Nelde HJ: Influence of nifedipine on stone formation and renal function in chlesterol-induced nephrolithiasis in rats. Urol Int 52: 87-92, 1994.

23. Ando R, Nagaya T, Suzuki S, Takahashi H, Kawai M, Okada A, Yasui T, Kubota Y, Umemoto Y, Tozawa K and Kohri K: Kidney stone formation is positively associated with conventional risk factors for coronary heart disease in Japanese men. J Urol 189: 1340-1346, 2013

24. Okada A, Yasui T, Hamamoto S, Hirose M, Kubota Y, Itoh Y Tozawa K, Hayashi Y and Kohri K: Genome-wide analysis of genes related to kidney stone formation and elimination in the calcium oxalate nephrolithiasis model mouse: Detection of stone-preventive factors and involvement of macrophage activity. J Bone Miner Res 24: 908-924, 2009.

25. Mantovani A, Sica A, Sozzani S, Allavena P, Vecchi A and Locati M: The chemokine system in diverse forms of macrophage activation and polarization. Trends Immunol 25: 677-686, 2004.

26. Zhang MZ, Yao B, Yang S, Jiang L, Wang S, Fan X, Yin H, Wong K, Miyazawa T, Chen J, et al: CSF-1 signaling mediates recovery from acute kidney injury. J Clin Invest 122: 4519-4532, 2012.

27. Lee S, Huen S, Nishio H, Nishio S, Lee HK, Choi BS, Ruhrberg C and Cantley LG: Distinct macrophage phenotypes contribute to kidney injury and repair. J Am Soc Nephrol 22: 317-326, 2011.

28. Takeuchi H, Tanaka M, Tanaka A, Tsunemi A and Yamamoto H: Predominance of M2-polarized macrophages in bladder cancer affects angiogenesis, tumor grade and invasiveness. Oncol Lett 11: 3403-3408, 2016.

29. Ichikawa J, Okada A, Taguchi K, Fujii Y, Zuo L, Niimi K, Hamamoto S, Kubota Y, Umemoto Y, Kohri K, et al: Increased crystal-cell interaction in vitro under co-culture of renal tubular cells and adipocytes by in vitro co-culture paracrine systems simulating metabolic syndrome. Urolithiasis 42: 17-28, 2014.

30. Taguchi K, Okada A, Kitamura H, Yasui T, Naiki T, Hamamoto S, Ando R, Mizuno K, Kawai N, Kohri K, et al: Colony-stimulating factor-1 signaling suppresses renal crystal formation. J Am Soc Nephrol 25: 1680-1697, 2014 